\title{
A INFLUÊNCIA DO TIPO DE SEDIMENTO E SUSBTRATO NA COMPOSIÇÃO DA COMUNIDADE DE MACROINVERTEBRADOS BENTÔNICOS
}

\author{
Fernanda Graziele Ciofi ${ }^{1}$ \\ Patrícia de Cássia Pereira ${ }^{2}$ \\ Daniele Padilha Cobra ${ }^{3}$
}

Marcos Vinicius Nunes ${ }^{4}$

RESUMO: Os tipos de sedimentos e substratos podem influenciar a composição da comunidade de macroinvertebrados bentônicos, em especial nos ecossistemas lênticos. O objetivo do estudo foi avaliar a relação do tipo de sedimento e substrato com a composição e distribuição da comunidade de macroinvertebrados bentônicos em um trecho da Represa de Furnas em Barranco Alto - Alfenas - MG. As amostras da comunidade de macroinvertebrados bentônicos foram coletadas em 4 pontos amostrais, feitos arrastos em uma área de $1 \mathrm{~m}$ da região litorânea, utilizando a D-net (rede em "D"). Em cada ponto de amostragem foram determinadas in situ as variáveis limnológicas e as concentrações de nutrientes. Foram determinados os índices de riqueza taxonômica; diversidade de Shannon-Wiener, equidade e dominância. Também foi determinada a similaridade de Bray-Curtis e aplicada a análise de correspondência Canônica (CCA). A maior riqueza taxonômica e abundância foram registradas no ponto $\mathrm{P} 3$, o qual apresenta substrato barroso e vegetação aquática. Enquanto, que os menores valores foram registrados no ponto $\mathrm{P} 1$, o qual possuía substrato arenoso e sem vegetação aquática, 
mostrando desta forma que o tipo de hábitat e substrato influenciam na composição e distribuição da comunidade de macroinvertebrados bentônicos.

Palavras-chave: Distribuição. Preferência. Micro-hábitat.

\section{INTRODUÇÃO}

Os tipos de sedimentos e substratos podem influenciar a composição da comunidade de macroinvertebrados bentônicos. Os fatores que influenciam a composição e distribuição da comunidade de macroinvertebrados bentônicos são os chamados fatores controladores (OLIVEIRA, 2009) entre eles: correnteza, tipo de substrato, presença de vegetação, temperatura, entre outros. Em ecossistemas lênticos o substrato é considerado um dos principais fatores, porém não é indicado analisá-lo sozinho (ESTEVES, 1998; HYNES, 2001).

De acordo com Hynes (2001) a comunidade de macroinvertebrados bentônicos compreende grupos de invertebrados com corpos superiores a 2 a $5 \mathrm{~mm}$, os quais habitam ou passam pelo menos parte do seu ciclo de vida enterrados ou sobre o substrato dos ecossistemas aquáticos continentais. Dentre os filos que compõe a comunidade de macroinvertebrados bentônicos podemos encontrar: Arthropoda (insetos, ácaros, crustáceos), Mollusca (gastrópodos e bivalves), Annelida (oligoquetos e hirudíneos), Nematoda, Platyhelminthes, entre outros (HAUER \& RESH 1996), constituindo um grupo diversificado de organismos, os quais habitam tanto ambientes lênticos (reservatórios, lagos e lagoas) como ambientes lóticos (rios, riachos e igarapés) (HAUER \& RESH, 1996; MERRITT \& CUMMINS, 1996).

Este grupo de organismos tem como característica o grande número de indivíduos, pertencentes à diferentes espécies, apresentando uma grande diversidade devido a sua história evolutiva e ao efeito multiplicador de nichos ecológicos (MARGALEF, 1983). Sendo esta diversidade, consequência da síntese de todos os fatores ambientais e interações ecológicas que influenciam uma assembléia de espécies co-ocorrentes (ALLAN, 1995). 
A distribuição, abundância e composição da comunidade de macroinvertebrados bentônicos estão relacionadas às características morfométricas e físico-químicas do habitat, à disponibilidade de recursos alimentares e ao hábito das espécies (RESH \& ROSENBERG, 1984; BOLTOVSKOY et al., 1995; MERRITT \& CUMMINS, 1996). Em especial nos ecossistemas lênticos, a distribuição e composição das comunidades de macroinvertebrados bentônicos, são influenciadas pelo tipo e composição do substrato. Uma vez que muitos táxons possuem certa preferência aos diferentes tipos de substrato (HYNES, 2001).

No entanto, estudos que visam relacionar a composição da comunidade de macroinvertebrados bentônicos com o tipo de substrato são escassos no Brasil, sendo a maioria realizados em regiões temperadas. Por isso este estudo tem o objetivo de avaliar a relação do tipo de sedimento e substrato com a composição e distribuição da comunidade de macroinvertebrados bentônicos em um trecho da Represa de Furnas em Barranco Alto - Alfenas - MG.

\section{METODOLOGIA}

As amostras da comunidade de macroinvertebrados bentônicos foram coletadas em 4 pontos amostrais, no dia 20 do mês de abril de 2013, cujas localizações e descrições estão na tabela 1.

Tabela 1 - Localização dos pontos amostrais, onde foram coletadas as amostras da comunidade de macroinvertebrados bentônicos na represa de Furnas em Alfenas - MG.

\begin{tabular}{l|l|l|l|l}
\hline Ponto de Amostragem & Latitude & Longitude & Tipo de substrato & Macrófitas \\
\hline Ponto 01 & $21^{\circ} 11.034^{\prime}$ & $45^{\circ} 57.385^{\prime}$ & areia & ausente \\
Ponto 02 & $21^{\circ} 10.984^{\prime}$ & $45^{\circ} 57.374^{\prime}$ & areia & presente \\
Ponto 03 & $21^{\circ} 10.902^{\prime}$ & $45^{\circ} 57.724^{\prime}$ & barro & presente \\
Ponto 04 & $21^{\circ} 10.912^{\prime}$ & $45^{\circ} 57.771^{\prime}$ & barro & ausente \\
\hline
\end{tabular}




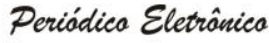

Para a coleta de macroinvertebrados bentônicos foram realizados arrastos em uma área de $1 \mathrm{~m}$ da região litorânea, utilizando a D-net (rede em "D") com abertura de malha de 250 micra. Em cada ponto de amostragem retiraram-se três amostras (pseudoréplicas), as quais foram analisadas conjuntamente. O material coletado foi fixado em formol $4 \%$, acondicionado em recipientes plásticos e transportado para o laboratório, onde foi lavado em água sobre peneiras de malhas de $1 \mathrm{~mm}$ e 0,212 $\mathrm{mm}$. Os animais retidos na peneira foram separados e fixados em etanol 70\% (SILVEIRA, 2004).

Os macroinvertebrados foram identificados sob microscópio estereoscópico, com o auxílio de literatura especializada: Pérez, (1988); Melo, (2003); García-Dávila \& Magalhães, (2003); Costa et al, (2004); Pes et al., (2005); Salles, (2006); Godoy, (2007) e Trivinho-Strixino, (2011). Os organismos bentônicos foram identificados até o menor nível taxonômico possível.

Em cada ponto de amostragem foram determinadas in situ as variáveis limnológicas (condutividade, oxigênio dissolvido, $\mathrm{pH}$ e temperatura) por meio do multisensor Horiba U10.

Para verificar se a diversidade de espécies é diferente entre os pontos amostrados foram calculados os seguintes índices: riqueza taxonômica; diversidade de ShannonWiener (H') (SHANNON \& WEAVER, 1949) e dominância. Também foi determinada a similaridade de Bray-Curtis entre a densidade dos organismos da comunidade bentônica nos pontos de amostragem. O índice de similaridade foi calculado utilizando o programa Past-Paleontological Statistics Software (HAMMER et al 2001). Foi também aplicada a análise de correspondência Canônica (CCA) através do programa CANOCO 3.12 (TER BRAAK \& SMILAUER, 2002), para verificar a relação entre sedimentos, substratos e formação da composição da comunidade bentônica.

\section{RESULTADOS E DISCUSSÃO}

Os resultados das variáveis abióticas (Tabela 2) mostraram que as maiores concentrações dos nutrientes foram registradas no ponto P3 (fosfato (orgânico, inorgânico e total dissolvido), nitrito e silicato) e P1 (fósforo total, nitrogênio total, nitrato e amônio). 
Tabela 2 - Valores das variáveis abióticas encontradas em todos os pontos amostrais, na represa de Furnas, em Barranco Alto - Alfenas - MG.

\begin{tabular}{l|c|c|c|c} 
Variáveis & P1 & P2 & P3 & P4 \\
\hline pH & 6.89 & 6.90 & 6.79 & $\mathbf{7 . 4 2}$ \\
Condutividade & 33 & $\mathbf{3 4}$ & 32 & 33 \\
Temperatura & 24.2 & 24.07 & 25.43 & $\mathbf{2 5 . 9 0}$ \\
Oxigênio dissolvido & 7.84 & $\mathbf{7 . 9 1}$ & 7.52 & 7.48 \\
Fósforo Total & $\mathbf{3 2 . 7 1}$ & 31.40 & 31.08 & 29.77 \\
Fosfato Total Dissolvido & 9.43 & 9.73 & $\mathbf{1 2 . 7 9}$ & 12.48 \\
Fosfato Orgânico & 2.92 & 3.23 & $\mathbf{4 . 2 3}$ & 4.22 \\
Fosfato Inorgânico & 6.51 & 6.51 & $\mathbf{8 . 5 6}$ & 8.27 \\
Nitrogênio Total & $\mathbf{1 2 6 . 2 2}$ & 122.96 & 89.69 & 64.91 \\
Nitrito & 0.02 & 0.13 & $\mathbf{0 . 3 0}$ & 0.07 \\
Nitrato & $\mathbf{6 5 . 5 7}$ & 50.93 & 23.54 & 15.99 \\
Amônio & $\mathbf{2 4 0 . 9 2}$ & 67.18 & 46.97 & 40.75 \\
Silicato & 6.33 & 4.34 & $\mathbf{7 . 4 3}$ & 5.49 \\
\hline
\end{tabular}

No total foram encontrados 15 táxons, sendo a maioria pertencentes à classe Insecta, representada por 10 táxons. E o único táxon que foi registrado em todos os pontos foi a subfamília Chironominae, conforme observado na tabela 2.

Tabela 3 - Riqueza taxonômica encontrada em todos os pontos amostrais, na represa de Furnas, em Barranco Alto - Alfenas - MG.

\begin{tabular}{|c|c|c|c|c|}
\hline Táxons & $\begin{array}{l}\text { Areia sem } \\
\text { vegetação }\end{array}$ & $\begin{array}{l}\text { Areia com } \\
\text { vegetação }\end{array}$ & $\begin{array}{l}\text { Barro com } \\
\text { vegetação }\end{array}$ & $\begin{array}{l}\text { Barro sem } \\
\text { vegetação }\end{array}$ \\
\hline \multicolumn{5}{|l|}{ Hydrachnidae } \\
\hline \multicolumn{5}{|l|}{ Dytiscidae } \\
\hline \multicolumn{5}{|l|}{ Elmidae } \\
\hline \multicolumn{5}{|l|}{ Hydrophilidae } \\
\hline \multicolumn{5}{|l|}{ Atrichopogon } \\
\hline \multicolumn{5}{|l|}{ Chironominae } \\
\hline \multicolumn{5}{|l|}{ Baetidae } \\
\hline \multicolumn{5}{|l|}{ Cyanallagma } \\
\hline \multicolumn{5}{|l|}{ Neogomphus } \\
\hline \multicolumn{5}{|l|}{ Cyrellus } \\
\hline \multicolumn{5}{|l|}{ Macrobachium jelskii } \\
\hline \multicolumn{5}{|l|}{ Macrobachium Petronioi } \\
\hline \multicolumn{5}{|l|}{ Corbicula flumínea } \\
\hline Pupa & & & & \\
\hline
\end{tabular}


A maior riqueza taxonômica foi registrada no ponto P3 (14 táxons), o qual apresenta substrato barroso e vegetação aquática. Neste mesmo local, também foi registrada o maior valor de abundância (193 indivíduos). Enquanto, que os menores valores de riqueza taxonômica (3 táxons) e abundância (4 indivíduos) foram registrados no ponto $\mathrm{P} 1$, o qual possuía substrato arenoso e sem vegetação aquática.

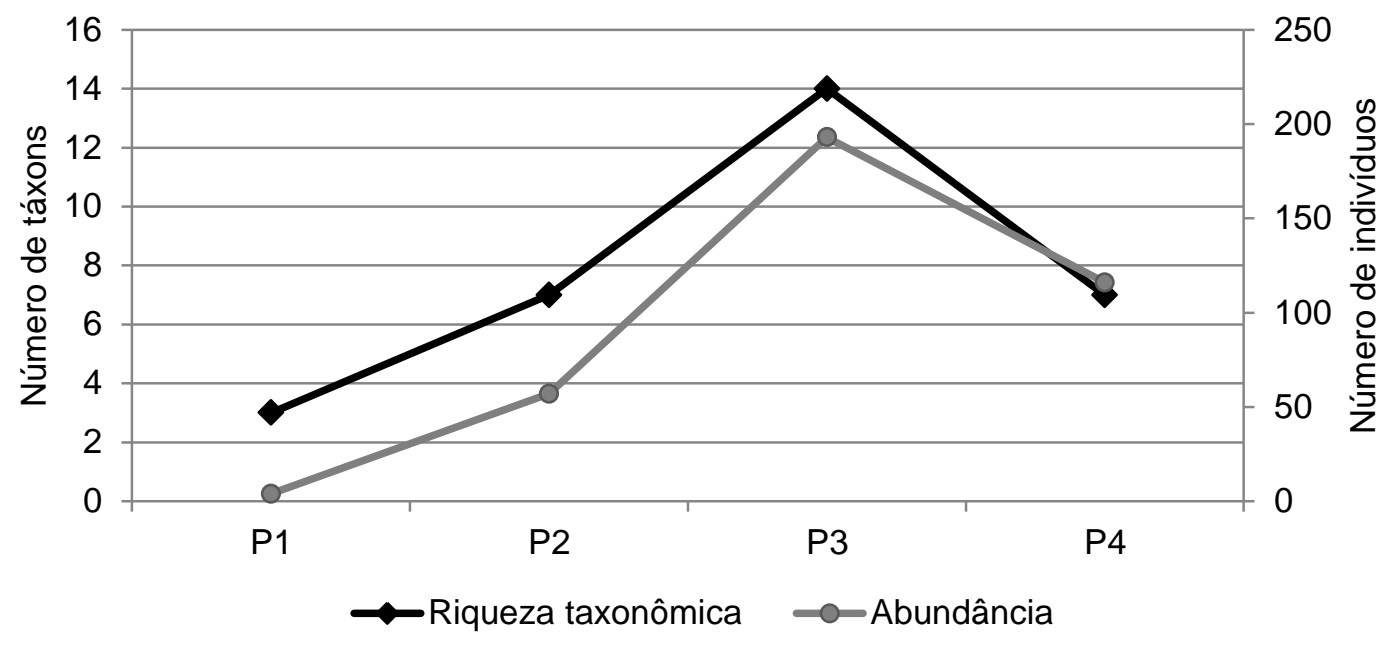

Figura 1 - Riqueza taxonômica e abundância absoluta encontradas em todos os pontos amostrais na represa de Furnas, em Barranco Alto - Alfenas - MG.

Da mesma forma que a riqueza, o maior valor do índice de diversidade foi registrado para o ponto $\mathrm{P} 3$ com 1,30 bits.ind $^{-1}$ e o menor valor foi no ponto P4 com 0,78 bits.ind ${ }^{-1}$ (Figura 2). No ponto P1 foram registrados o menor valor do índice de dominância $(0,37)$ e o maior do índice de equidade $(0,94)$. Estes dados são consequência da baixa riqueza e abundância no P1. Enquanto que no P4 apesar de terem sido registrados os segundos maiores valores de riqueza e abundância, neste ponto foram registrados 0 maior valor do índice de dominância $(0,65)$ e o menor do índice de equidade $(0,40)$. Este resultado se deve ao alto valor de abundância de Chironominae, ao se comparar com a abundância dos demais táxons. 


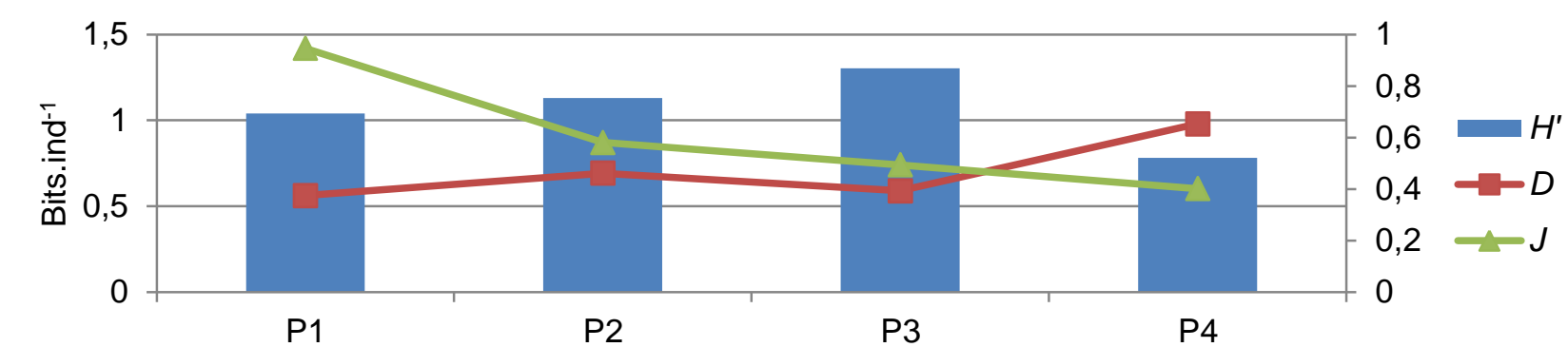

Figura 2 - Valores dos índices de Diversidade Shannon-Wiener $(H)$, Equidade $(\mathcal{J})$ e Dominância $(D)$ encontradas nos pontos amostrais na represa de Furnas, em Barranco Alto - Alfenas - MG.

Conforme é possível observar na figura 3, a qual representa o dendrograma de similaridade (Bray-Curtis), os pontos com maior similaridade foram P3 e P4 com um pouco mais de $60 \%$ de semelhança entre si. Enquanto que estes dois pontos apresentaram baixa semelhança com o ponto P2 $(<20 \%)$ e com o ponto P1 $(<10 \%)$.

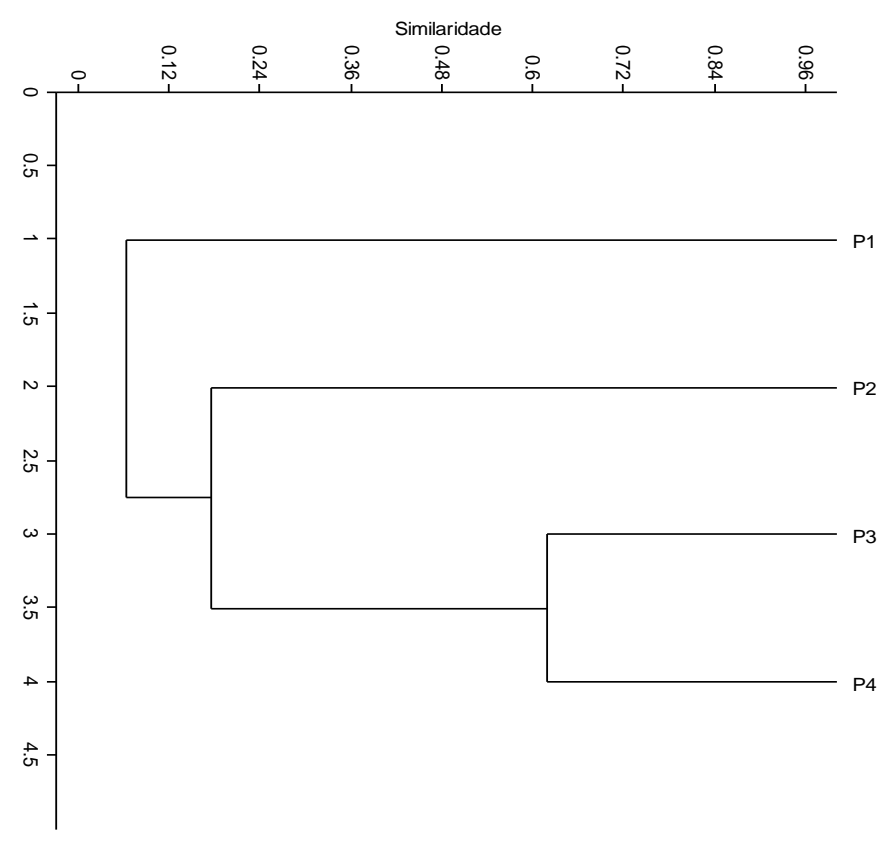

Figura 3 - Dendrograma de similaridade de Bray-Curtis para os pontos amostrais na represa de Furnas, em Barranco Alto - Alfenas - MG. Cf $=0.9839$ 
A Figura 4 é composta pelos resultados obtidos para a análise de correspondência canônica (CCA) entre a abundância dos organismos da comunidade de macroinvertebrados bentônicos e as variáveis ambientais nos pontos amostrados na represa de Furnas, em Barranco Alto - Alfenas - MG.

A porcentagem total explicada pelos dois primeiros componentes na análise de correspondência canônica (CCA) para a abundância dos principais grupos da comunidade de macroinvertebrados bentônicos e as variáveis ambientais foi de $85 \%$, e indicou que nenhuma variável foi significativa. A CCA também indicou que os maiores valores de fosfato (orgânico, inorgânico e total dissolvido), silicato e nitrito correlacionaram com ponto P3 da mesma maneira que a maioria dos táxons encontrados correlacionaram a este ponto, como: Coleoptera, Odonata e Ephemeroptera.

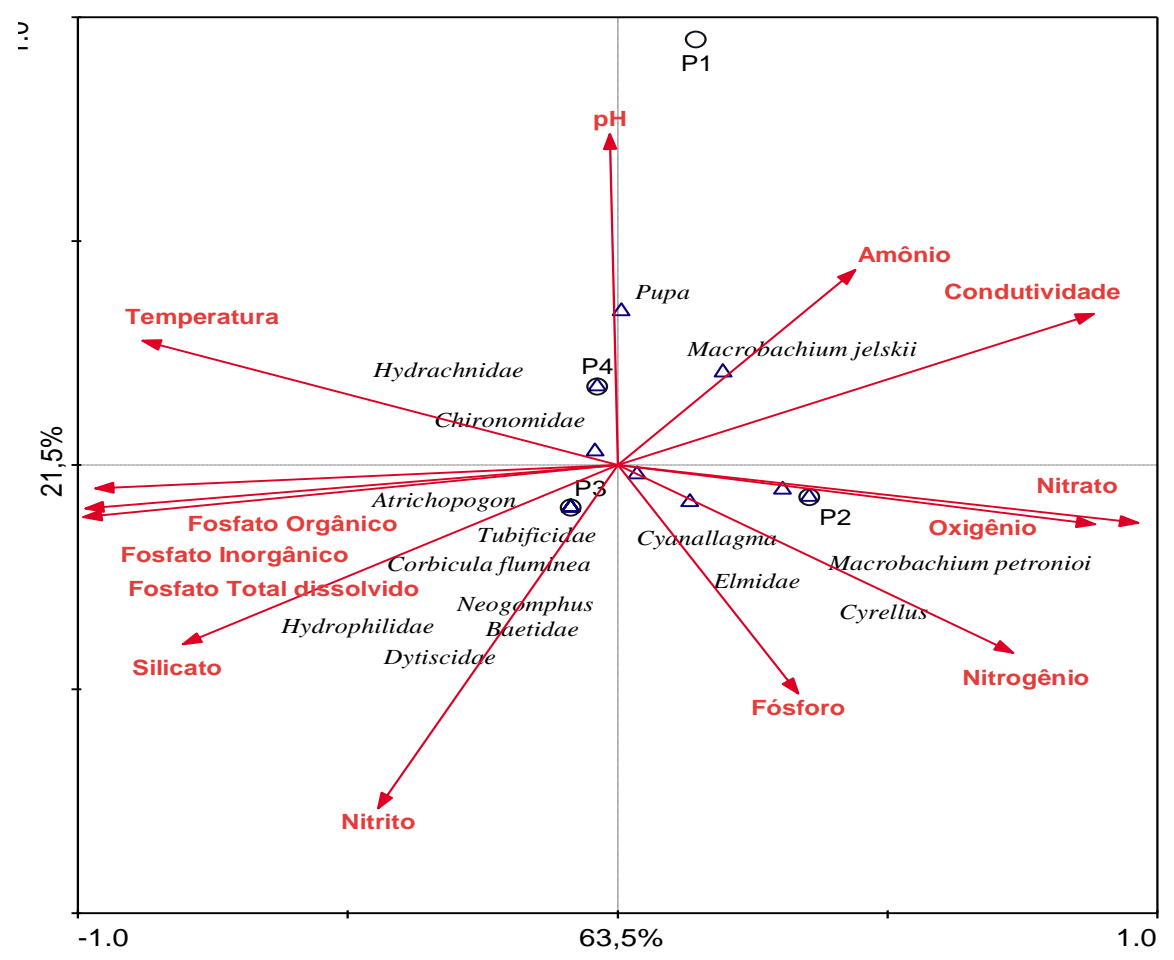

Figura 4 - Diagrama de ordenação CCA da abundância dos principais grupos da comunidade de macroinvertebrados bentônicos com as variáveis abióticas nos pontos amostrais na represa de Furnas, em Barranco Alto - Alfenas - MG.

Segundo Goulart \& Callisto (2003) a distribuição e diversidade de macroinvertebrados são diretamente influenciadas pelo tipo de substrato, morfologia do ecossistema, quantidade e tipo de detritos orgânicos, presença de vegetação aquática, 
presença e extensão de matas ciliar, e indiretamente afetados por modificações nas concentrações de nutrientes e mudanças na produtividade primária.

Os maiores valores de riqueza, abundância e diversidade encontrados no ponto P3, estão relacionados às características da região amostrada. Uma vez que, o substrato influencia diretamente sobre a abundância e diversidade da comunidade de macroinvertebrados bentônicos, visto que, o tamanho das partículas, a presença de matéria orgânica e a textura da superfície são fatores importantes para a sua composição e distribuição (ALLAN, 2001).

Segundo Benke et al. (1984) sistemas aquáticos com substrato arenoso apresentam menor biomassa de macroinvertebrados bentônicos do que ambientes com substrato lodosos e com a presença de matéria orgânica, raízes, macrófitas e seixos. Estes fatos foram observados neste estudo, pois o ponto P3 além de ter substrato com característica barroso apresentava a presença de vegetação aquática. Ao contrário, o ponto $\mathrm{P} 1$, no qual foram registrados os menores valores de riqueza e densidade, possuía substrato arenoso e sem a presença de vegetação aquática. Isto porque em substratos arenosos é esperado menores valores de abundância, por causa da instabilidade deste substrato e por sua baixa quantidade de matéria orgânica, levando à baixa oferta de alimento (BAPTISTA et al., 2000).

Esta maior riqueza e diversidade registrada para o ponto P3 mostra como as características do ambiente influenciam na diversidade e abundância da comunidade de macroinvertebrados bentônicos. Visto que, muitos táxons possuem certa preferência aos diferentes tipos de substrato (HYNES, 2001). E a riqueza e a diversidade estão relacionadas com a heterogeneidade e complexidade de hábitats (VINSON \& HAWKINS, 1998). Sendo que a diversidade e abundância tendem a aumentar com a estabilidade do substrato e a presença de detritos orgânicos (ALLAN, 1995).

Além disso, a presença de macrófitas aquáticas é responsável por proporcionar uma maior heterogeneidade ambiental e uma maior diversidade e disponibilidade de alimento, contribuindo para o aumento da diversidade. Isto porque ambientes heterogêneos proporcionam uma maior quantidade de micro-habitats e de microclimas, possibilitando que diferentes espécies possam coexistir, consequentemente aumentando a riqueza taxonômica do local (TOWNSEND, 2006). E ambientes taxonomicamente diversos, tendem possuir espécies pouco abundante (ODUM, 1988). 


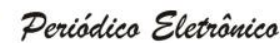

\section{CONCLUSÃO}

Os resultados mostraram que o tipo de hábitat e substrato influenciam na composição e distribuição da comunidade de macroinvertebrados bentônicos. A influência do tipo de substrato pode ser observada, ao verificar uma maior diversidade taxonômica e abundancia nos pontos com sedimento barroso em comparação dos pontos com sedimento arenoso. Da mesma forma os pontos com a presença de vegetação aquática foram taxonomicamente mais diversos em relação aos que eram desprovidos de vegetação aquática.

\section{AGRADECIMENTOS}

Os autores agradecem ao Departamento de Ecologia e Biologia Evolutiva da Universidade federal de São Carlos, em especial a prof $^{a}$ Dr. Odete Rocha e ao Dr. José Valdecir de Lucca, pelas realizações das análises dos nutrientes e empréstimo do material de campo.

\section{REFERENCIAS}

ALLAN, J.D. Stream Ecology: structure and function of running waters. London: Chapman \& Hall, 388p. 1995.

BAPTISTA, D.F.; BUSS, D.F.; DROVILLÉ, L.F.M. \& NESSIMIAN, J.L. Diversity and habitat preference of aquatic insects along the longitudinal gradient of the Macaé river basin, Rio de Janeiro, Brazil. Revista Brasileira de Biologia, 61: 249-258. 2000.

BENKE, A.C. Secondary production of aquatic insects. In: RESH, V.H. \& ROSENBERG, D.M. The ecology of aquatic insects. New York: Praeger Publishers, 1984.

BOLTOVSKOY, D.; TELL ,G. \& DADON, R. Afinidad entre comunidades bentonicas de un ambiente lotico, p. 203-214. In: LOPRETTO, E.C. \& TELL, G. (Eds). Ecosistemas de aguas continentales: metodologias para su estudio. Argentina, Ed. Sur, Tomo I, 376p. 1995. 
COSTA, J.M.; SOUZA, L.O.I. \& OLDRINI, B.B. Chave para identificação das famílias e gêneros das larvas conhecidas de Odonata do Brasil: comentários e registros bibliográficos (Insecta, Odonata). Publicações avulsas do Museu Nacional, 99: 1 - 44. 2004.

ESTEVES, F.A. Fundamentos de limnologia. 2. ed. Rio de Janeiro: Interciência. 1998.

GARCÍA-DÁVILA, C.R. \& MAGALHÃES, C. Revisão taxonômica dos camarões de água doce (Crustacea: Decapoda: Palaemonidae, Sergestidae) da Amazônia Peruana. Acta Amazonica, 33: 663-686. 2003.

GODOY, B.S. Estrutura da assembleia de Gerromorpha (Heteroptera) em igarapés dos municípios de Rio Preto da Eva e Manaus, Amazonas. Dissertação (Mestrado). Instituto Nacional de Pesquisa da Amazonas - INPA/UFAM, Manaus. 2007.

GOULART, M. \& CALLISTO, M. Bioindicadores de qualidade de água como ferramenta em estudos de impacto ambiental. Revista da FAPAM, 2: 153 - 164, 2003.

HAMMER, O.; HARPER, D.A.T. \& RYAN, P.D. PAST: Palaeontological Statistics Software Package for education and data analysis. Palaeontologia Electronica, v. 4, n. 1, 9p. 2001. Disponível em < http://folk.uio.no/ohammer/past >

HAUER, F.R. \& V.H. RESH. Benthic macroinvertebrates, p. 339-369. In: HAUER, F.R. \& LAMBERTI, G.A. (Eds). Stream ecology. San Diego, Academic Press, 674p. 1996.

HYNES, H.B. The Ecology of Running Waters. Ontaro: The blackburn press. 555 p. 2001.

MARGALEF, R. Limnologia. Barcelona. Ediciones Omega. 1983.

MELO, G.A.S. Manual de identificação dos Crustacea Decapoda de água doce do Brasil. São Paulo: Edições Loyola. 2003.

MERRIT, R. \& CUMMINS, K. An introduction to the aquatic insects of North America. 3 ed. Kendall: Hunt Publishing. 1996.

ODUM, E.P. Ecologia. Rio de Janeiro: Editora Guanabara Koogan. 1988.

OLIVEIRA, P.C.R. Comunidade de macroinvertebrados bentônicos e qualidade da água e do sedimento das bacias hidrográficas dos Rios Lavapés, Capivara, Araquá e Pardo, Município de Botucatu(SP) e região. (Dissertação de Mestrado) Universidade Estadual Paulista, Botucatu. 2009.

PÉREZ, G.R. Guia para el estúdio de los macroinvertebrados acuáticos del Departamento de Antioquia. Fondo Fen Colombia, Conciencias, Universidad de Antioquia, Bogotá. 217p. 1988. 
PES, A.M.O., HAMADA, N. \& NESSIMIAN, J.L. Chaves de identificação de larvas para as famílias e gêneros de Trichoptera (Insecta) da Amazônia Central, Brasil. Revista brasileira de entomologia, 49: 181-204. 2005.

RESH, V.H. \& ROSENBERG, D.M.. The ecology of aquatic insects. New York, Praeger Publishers, 625p. 1984.

SALLES, F.F. A ordem ephemeroptera no Brasil (Insecta): taxonomia e diversidade. Tese (Doutorado). Universidade Federal de Viçosa, Viçosa. 2006.

SHANNON, C.E. \& WEAVER, W. The mathematical theory of communication. University of Illinois, Press: Urbana. 1949.

SILVEIRA, M.P.; QUEIROZ, J.P. \& BOEIRA, R.C. Protocolo de coleta e preparação de amostras de macroinvertebrados bentônicos em riachos. Comunicado técnico. Embrapa Meio Ambiente, Jaguariúna, 2004.

TER BRAAK, C.J.F. Canonical Correspondence Analysis: a new eigenvector technique for multivariate direct gradient analysis. Ecology, 67: 1167-1179. 1986.

TRIVINHO-STRIXINO, S. Larvas de Chironomidae. Guia de identificação e diagnose dos gêneros. São Carlos, Depto Hidrobiologia/Lab. Entomologia Aquática/UFSCar, 2011.

TOWNSEND, C.R.; BEGON, M. \& HARPER, J.L. Fundamentos em ecologia. Artmed, Porto Alegre. 2006.

VINSON, M.R \& HAWKINS, C.P. Biodiversity of stream insects variation at local, basin and regional scales. Annual Revista Entomologia. 43: 271-293. 1998. 